Low triiodothyronine concentration in preterm infants $w$ as correlated with a mean 6.6 point deficit in overall IQ scores ( 8.5 deficit on verbal scale) on the WISC scales at 8-year follow up in a study of 236 infants examined at the Infant and Child Nutrition Group, Medical Research Council Dunn Nutrition Unit, Cambridge, UK. (Lucas A, Morley R, Fewtrell MS. BMI 4 May 1996;312:1132-3). Brook CGD, of Cobbold Laboratories, Middlesex Hospital, London, in a Commentary: Do preterm infants need thyroxine replacement? (BMI 1996;312:1133) cautions that thyroxine therapy for hypothyroid mothers is unlikely to benefit premature babies, and triiodothyronine replacement in premature infants may be dangerous. Neonatologists generally withhold therapy pending a retest after the infant reaches term. An increased risk of developing cerebral palsy and cognitive deficits has been reported in premature infants with low thyroxine levels in the first week of life (N Engl I Med March 28 1996).

Iodine deficiency diseases (IDD), a major international public health problem especially affecting developing countries, may be prevented by the administration of iodinized salt. My colleague, Dr Charles Swisher, provided me with several references to the topic, pointing out that estimates have shown 200 million affected by IDD and 800 million people at risk worldwide for IDD, a total of "1 billion brains" at risk of maldevelopment or malfunction! The most serious complication of IDD is endemic cretinism, but milder forms of IDD may be associated with impaired cognitive function and learning disabilities in later childhood. For further reviews of IDD, see Delange F. Thyroid Spring 1994;4:107-128; Lamberg BA. Ann Med Oct 1991;23:367-372; Hetzel BS et al. Neuropath \& Applied Neurobiol 1988;14:93-104; Maberly GF. I Nutrition Aug 1994;124(8 Suppl):1473-78S.

\title{
ATTENTION DEFICITS AND CONGENITAL HYPOTHYROIDISM
}

The ability to sustain attention was studied using continuous performance tasks in 48 children with early treated congenital hypothyroidism $(\mathrm{CH})$ and 35 healthy controls at the University of Groningen, The Netherlands. In 38 patients with T4 levels $<50 \mathrm{nmol} / 1$ as neonates, performance of a computer-paced task declined over time, suggesting impairment of sustained attention. In a self-paced task, an initial performance decline was followed by an improvement in the final stages, this pattern being most pronounced in the low T4 group, reflecting a greater performance variability over time and a problem with sustained attention. The performance of the 10 children with intermediate T4 levels $(=/>50 \mathrm{nmol} / 1$ as neonates) fell between the control group and the low T4 group. The performance declines were correlated with intelligence, but the difference in performance decline between the low T4 group and controls was not related to intelligence. No correlation was found between onset of treatment for $\mathrm{CH}$ and sustained attention. A suboptimal motor system may have been a factor underlying the sustained attention deficit in $\mathrm{CH}$ children. (Kooistra L et al. Sustained attention problems in children with early treated congenital hypothyroidism. Acta Paediatr April 1996;85:425-9). (Respond: Dr L Kooistra, University of Groningen, Laboratory of Experimental Clinical Psychology, Grote Kruisstraat 2/1, 9712 TS Groningen, The Netherlands).

COMMENT. Motor, cognitive, and motivational factors may influence scores on continuous performance tasks. All three factors can be important influences on sustained attention in children with congenital hypothyroidism. 
Pituitary enlargement on MRI in congenital hypothyroidism, mimicking a sellar or suprasellar tumor, is reported from the Bai Jerbai Wadia Hospital for Children and Institute of Child Health and Research Centre, Parel, Bombay 400 012, India. (Desia MP et al. Arch Pediatr Adolesc Med June 1996;150:623-628). The $\mathrm{CH}$ was long-standing and untreated. MRI was abnormal in all patients. One child presented with acute headache, vomiting, and papilledema. MRI showed pituitary enlargement, suggesting a pituitary adenoma. Bone age was retarded. Symptoms improved within 10 days of levothyroxine therapy, and an MRI at 2 months showed marked regression of pituitary size. Serum triiodothyronine and thyroxine levels were low, and thyrotropin was elevated in all patients. Prolactin was elevated in 5 patients and was highest in the child with neurologic symptoms. This complication of congenital hypothyroidism will be encountered primarily in developing countries and is rare in the US, since most patients will have received treatment soon after birth. However, an awareness of pituitary enlargement as a sign of $\mathrm{CH}$ will avoid unnecessary neurosurgical intervention in children with untreated or subtle hypothyroidism.

\section{TOXIC DISORDERS}

\section{COCAINE-INDUCED HORMONAL AND BEHAVIORAL CHANGES}

Behavioral and hormonal responses in 30 preterm cocaine-exposed infants were compared with a cohort of 30 non-cocaine-exposed preterm infants at the Touch Research Institute, University of Miami School of Medicine, FL. Cocaine-exposed infants had smaller head circumference at birth, longer stays in the intensive care unit, a higher incidence of intraventricular hemorrhage, inferior performance on the Brazelton Neonatal Behavioral Assessment Scale (range of state, regulation of state, and depression clusters), decreased periods of quiet sleep, and increased levels of agitated behavior, including tremulousness, limb movements, and clenched fists. They also had higher urinary norepinephrine, dopamine, and cortisol levels and lower plasma insulin levels than controls. Epinephrine and glucose levels were unchanged. (Scafidi FA, Field TM et al. Cocaine-exposed preterm nenates show behavioral and hormonal differences. Pediatrics June 1996;97:851-855). (Reprints: Dr Tiffany M Field, Touch Research Institute, University of Miami School of Medicine, PO Box 016820, Miami, FL 33101).

COMMENT. Cocaine-exposed infants require careful follow-up for early diagnosis and therapy of neurobehavioral complications. A frequent history of prenatal cocaine exposure in foster children with attention deficit hyperactivity disorders is of interest in relation to the changes in catecholamine metabolism noted in the above study. For reference to neurological correlates of fetal cocaine exposure, see Ped Neur Briefs Feb 1996;10:9-10; and Progress in Pediatric Neurology I and II PNB Publishers, 1991 and 1994.

Other prenatal toxic factors that may underly cognitive, behavioral, and attentional deficits in childhood include alcohol, PCBs, and nicotine.

Fetal alcohol syndrome and ophthalmological abnormalities are reported in 25 children examined at Children's Hospital, Goteberg, Sweden. (Stromland K, Hellstrom A. Pediatrics June 1996;97:845-850). The fundus was abnormal in 23, of whom 19 had optic nerve hypoplasia. Concomitant strabismus occurred in 13. Other abnormalities included microphthalmos, 